\title{
Influence of Transistor Parameters on the Noise Margin of Organic Digital Circuits
}

\author{
Stijn De Vusser, Student Member, IEEE, Jan Genoe, Member, IEEE, and Paul Heremans
}

\begin{abstract}
The concept of noise margin is crucial in the design and operation of digital logic circuits. Analytical expressions for the transfer curves of an inverter based on two depletion-mode p-type organic thin-film transistors (OTFTs) were calculated. Based on these expressions, the values for the noise margin of organic-based inverters were calculated. In this paper, the influence of the OTFT parameters on the noise margin is presented. Knowing that statistical variations of the transistor parameters are inherent to OTFT technology, these statistical variations are also taken into account. Finally, a circuit yield analysis is presented.
\end{abstract}

Index Terms-Digital circuit robustness, noise margin, organic thin-film transistors (OTFTs), yield.

\section{INTRODUCTION}

$\mathbf{O}$ RGANIC electronics is an area of considerable interest. Although a lot of research is still going on, the first commercialized products are already available in the market. Display applications based on organic light-emitting diodes (OLEDs) are an important application, especially if they can be driven by a backplane using organic thin-film transistors (OTFTs). Another important driver in the field of organic electronics is the development of an organic radio frequency identification (RFID) tag. It is clear that OTFTs are expected to be used in digital circuits.

To ensure the proper operation of the digital circuit, one has to take into account some stability considerations. In particular, the noise margin $(N M)$ of an inverter is an important figure for the stability of the digital circuits. The concept of the noise margin was developed by Hill in the late 1960s [1], [2]. Noise margin was originally defined as "the maximum allowable spurious signal that can be accepted by a device when used in a system while still giving correct operation."

For an inverter, it is possible to define the high and low voltages $V_{\mathrm{OH}}$ and $V_{\mathrm{OL}}$, where $V_{\mathrm{OH}}$ is the minimum output high voltage and $V_{\mathrm{OL}}$ is the maximum output low voltage. Furthermore, one can define the transition points $V_{\mathrm{IH}}$ and $V_{\mathrm{IL}}$,

Manuscript received August 4, 2005; revised December 29, 2005. This work was supported in part by the EU-funded Integrated Project PolyApply (IST 507143) (http://www.polyapply.org). The review of this paper was arranged by Editor J. Kanicki.

S. De Vusser and P. Heremans are with the Interuniversity Microelectronics Center (IMEC), B-3001 Leuven, Belgium and also with the Electrical Engineering Department, Katholieke Universiteit Leuven, B-3001 Leuven, Belgium (e-mail: stijn.devusser@imec.be).

J. Genoe is with Interuniversity Microelectronics Center (IMEC), B-3001 Leuven, Belgium.

Digital Object Identifier 10.1109/TED.2006.870876 where $V_{\mathrm{IH}}$ is the minimum input high voltage that can be treated as a high voltage at the input of an inverter and $V_{\mathrm{IL}}$ is the maximum input low voltage that can be treated as a low voltage at the input of an inverter. If one has an inverter satisfying the relationships

$$
\begin{aligned}
V_{\text {in }} \leq V_{\mathrm{IL}} & \Rightarrow V_{\text {out }} \geq V_{\mathrm{OH}} \\
V_{\text {in }} \geq V_{\mathrm{IH}} & \Rightarrow V_{\text {out }} \leq V_{\mathrm{OL}} \\
V_{\mathrm{IH}} & >V_{\mathrm{IL}}
\end{aligned}
$$

with $V_{\text {in }}$ being the input voltage and $V_{\text {out }}$ the output voltage of the inverter, then the high- and low-state noise margins $\left(N M_{H}\right.$ and $N M_{L}$, respectively) can be defined mathematically as

$$
\begin{aligned}
N M_{H} & =V_{\mathrm{OH}}-V_{\mathrm{IH}} \\
N M_{L} & =V_{\mathrm{IL}}-V_{\mathrm{OL}} .
\end{aligned}
$$

To ensure a stable operation of the inverter, its noise margins $N M_{H}$ and $N M_{L}$ need to be sufficiently positive. The noise margin of a logic gate is directly related to the reliable and robust operation of the logic circuit: the higher the noise margin, the more reliable the circuit. As the circuit complexity (expressed by the number of transistors in a circuit) increases, the noise margin can even be related to circuit yield. To the best of our knowledge, only a limited number of publications specifically deal with the noise margin and yield aspects of OTFT-based inverters [3]-[5].

If one considers a chain of identical inverters, some inverter $M$ may deliver a high logical state at its output, $V_{\text {out }, M}$. If the noise voltage at the input of the next inverter $M+1$ in the chain exceeds $N M_{H}$, inverter $M+1$ may treat the combination of noise and $V_{\text {out, } M}$ as a low logical state voltage, producing a high logical state at its output $V_{\text {out }, M+1}$. This causes an incorrect operation of the circuit, because one expects the output of the inverter $M+1$ to be in the low logical state.

Similarly, some inverter $M$ in a chain may deliver a low logical state at its output $V_{\text {out, } M}$. If the noise voltage at the input of the inverter $M+1$ exceeds $N M_{L}$, inverter $M+1$ may treat the combination of noise and $V_{\text {out }, M}$ as a high logical state voltage, producing a low logical state at its output, $V_{\text {out }, M+1}$. Again, this causes an incorrect operation of the circuit, because one expects the output of the inverter $M+1$ to be in the high logical state.

Equations (1) and (2) define forbidden regions for inverter transfer curves. The gray areas in Fig. 1 graphically represent 


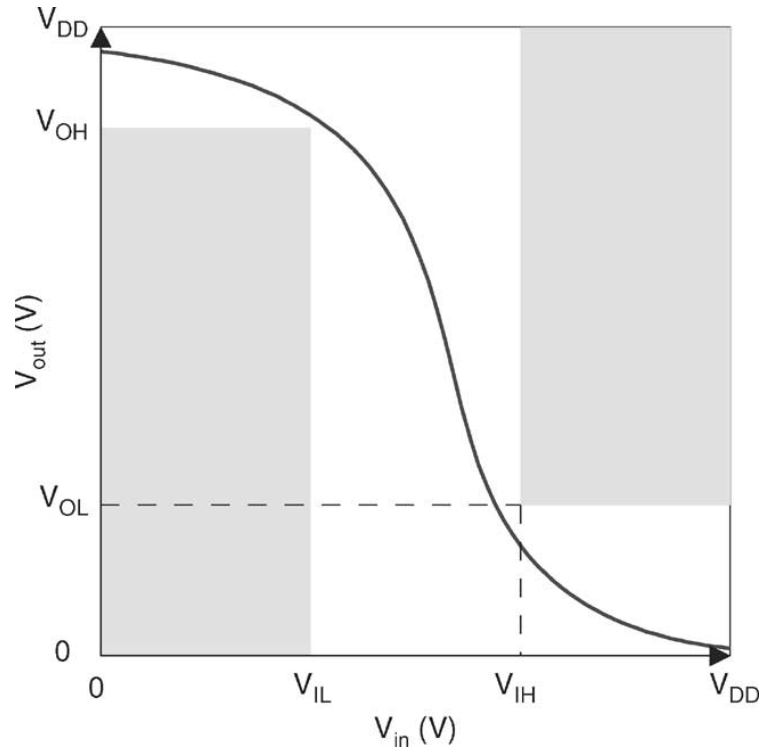

Fig. 1. Inverter transfer curve and the forbidden regions defined by (1) and (2).

all combinations of $V_{\text {in }}$ and $V_{\text {out }}$ that are forbidden by (1) and (2). Equations (4) and (5) represent a lower limit for the noise margins of any inverter curve that avoids the forbidden regions. Indeed, an inverter transfer curve that does not have a single point in common with the gray areas, such as the one shown in Fig. 1, will actually have higher noise margins than the ones given by (4) and (5).

\section{Definition of the Noise MARgin}

Whereas the definition of the noise margin is quite straightforward, different concepts have been proposed to extract the points $V_{\mathrm{OH}}, V_{\mathrm{OL}}, V_{\mathrm{IH}}$, and $V_{\mathrm{IL}}$ from a given inverter transfer curve. Hence, several methods exist to calculate the value of the noise margin, yielding remarkably different results in certain cases. An excellent overview of several concepts can be found in the literature [6], [7].

Because of its solid theoretical basis, we have chosen the maximum equal criteria (MEC) in which one has to maximize the area of a square that fits in the inverter transfer curve loops $V_{\text {out }}=f\left(V_{\text {in }}\right)$ and $V_{\text {out }}=f^{-1}\left(V_{\text {in }}\right)$. Both the high- and lowstate noise margins are equal to the side of the square. Fig. 2 illustrates the concept of the MEC in order to calculate a value for the noise margin for a given inverter transfer curve.

\section{Calculation of the Noise Margin}

Although complementary logic has been demonstrated using organic semiconductors [8]-[11], most inverters reported in the literature use a p-type-only architecture [12]-[14]. Pentacene is the benchmark semiconducting material for organic electronic circuits. Pentacene OTFTs often behave as depletionmode p-type transistors. Hence, our calculations are based on an inverter architecture using two p-type OTFTs, each with a positive threshold voltage $V_{T}$.

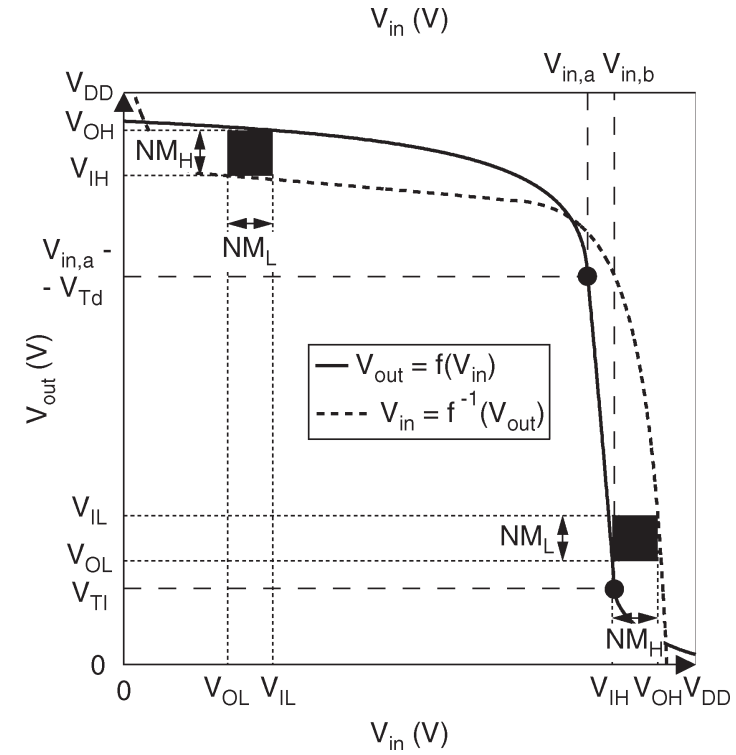

Fig. 2. Graphical illustration of the high- and low-state noise margins $N M_{H}$ and $N M_{L}$ as defined by the MEC. The dots represent the position of $V_{\text {in }, a}$ and $V_{\text {in }, b}$.

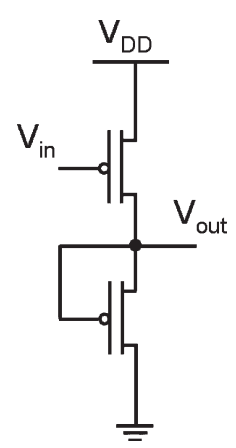

Fig. 3. Architecture of the inverter favored for p-type transistors with positive $V_{T}$.

In a p-type-only technology, it is favored that the drain-source current of the load transistor be kept constant as much as possible. This can be done by connecting the gate of the load transistor to its source, leading to the architecture in Fig. 3. This architecture is known as the depletion-load MOS inverter and has been extensively studied in Si MOS circuit design [15]. It only works using two depletion-mode transistors: If the threshold voltage of the load transistor $V_{\mathrm{Tl}}$ was negative, then $\left|V_{\mathrm{GS}, 1}\right|<\left|V_{\mathrm{Tl}}\right|$. In that case, the load transistor, acting as a current source, would either be completely off or operate in the subthreshold regime. In both cases, it only produces a very low current. Obviously, the operation of the inverter is severely influenced by this.

The depletion-load MOS inverter yields a relatively high gain as compared to the enhancement-load MOS inverter, another well-known MOS inverter architecture. Although there are some tradeoffs in terms of speed between the depletion- and the enhancement-load MOS inverter, the higher gain of the former justifies why it is nowadays very common in an OTFT-based circuit design. The calculations in this paper are based on this architecture. 
During the design of our inverters, we have chosen to take the $W / L$ of the load transistor five times as large as the $W / L$ of the driver transistor $\left(n=\left((W / L)_{L} /(W / L)_{D}\right)=5\right)$.

We have modeled our OTFTs using the basic transistor equations [16], in which the drain-source current in the linear regime (i.e., $\left|V_{\mathrm{DS}}\right| \leq\left|V_{\mathrm{GS}}-V_{T}\right|$ ) is given by

$$
I_{\mathrm{DS}}=-\frac{W}{L} \mu C_{\mathrm{ox}}\left(V_{\mathrm{GS}}-V_{T}-\frac{V_{\mathrm{DS}}}{2}\right) V_{\mathrm{DS}} .
$$

The drain-source current in the saturation regime (i.e., $\left.\left|V_{\mathrm{DS}}\right| \geq\left|V_{\mathrm{GS}}-V_{T}\right|\right)$ is given by

$I_{\mathrm{DS}}=-\frac{1}{2} \frac{W}{L} \mu C_{\mathrm{ox}}\left(V_{\mathrm{GS}}-V_{T}\right)^{2}\left(1-\lambda\left(V_{\mathrm{DS}}-\left(V_{\mathrm{GS}}-V_{T}\right)\right)\right)$.

Starting from (6) and (7), we have calculated the analytical expressions for the inverter transfer curve. We have distinguished four operation regimes for the inverter, based on the values of four parameters $V_{\mathrm{in}, a}, V_{\mathrm{in}, b}, V_{\mathrm{in}, c}$, and $V_{\mathrm{in}, d}$.

In the mathematical derivations throughout this paper, $V_{\mathrm{in}, a}$ represents the input voltage at which the driving transistor goes from the linear into the saturation regime, whereas the load transistor remains in the saturation regime. Correspondingly, $V_{\mathrm{in}, b}$ is the input voltage at which the load transistor goes from the saturation into the linear regime, whereas the driving transistor remains in the saturation regime. Fig. 2 shows the position of $V_{\mathrm{in}, a}$ and $V_{\mathrm{in}, b}$ as well as the graphical illustration of their mathematical definitions. The mathematical definitions are given as

$$
\begin{aligned}
V_{\mathrm{out}, a} & =f\left(V_{\mathrm{in}, a}\right) \\
& =f_{\mathrm{LS}}\left(V_{\mathrm{in}, a}\right)=f_{\mathrm{SS}}\left(V_{\mathrm{in}, a}\right) \\
& =V_{\mathrm{in}, a}-V_{\mathrm{Td}} \\
V_{\mathrm{out}, b} & =f\left(V_{\mathrm{in}, b}\right) \\
& =f_{\mathrm{SS}}\left(V_{\mathrm{in}, b}\right)=f_{\mathrm{SL}}\left(V_{\mathrm{in}, b}\right) \\
& =V_{\mathrm{Tl}} .
\end{aligned}
$$

In these definitions, $f_{\mathrm{LS}}\left(V_{\mathrm{in}}\right), f_{\mathrm{SS}}\left(V_{\mathrm{in}}\right)$, and $f_{\mathrm{SL}}\left(V_{\mathrm{in}}\right)$ are the applicable expressions for the inverter transfer curve in the range of interest. A more formal definition of these expressions will be given later on. $V_{\mathrm{Td}}$ and $V_{\mathrm{Tl}}$ represent the threshold voltage of the driver and the load transistors, respectively. Analytical expressions for $V_{\mathrm{in}, a}$ and $V_{\mathrm{in}, b}$ are found by solving (8)-(13) and are given by (30) and (31) in the Appendix, respectively.

Usually, $V_{\mathrm{in}, a}<V_{\mathrm{in}, b}$. In this case, the following operation regimes apply for the inverter transfer curve.

1) $0 \leq V_{\text {in }} \leq V_{\text {in }, a}$ : The driving transistor is in the linear regime; the load transistor is in the saturation regime. The analytical expression for the inverter transfer curve in this region is given by $f_{\mathrm{LS}}\left(V_{\mathrm{in}}\right)(26)$ in the Appendix.
2) $V_{\text {in, } a} \leq V_{\text {in }} \leq V_{\text {in }, b}$ : The driving and the load transistors are both in the saturation regime. The analytical expression for the inverter transfer curve in this region is given by $f_{\mathrm{SS}}\left(V_{\text {in }}\right)(27)$ in the Appendix.

3) $V_{\text {in }, b} \leq V_{\text {in }} \leq V_{\mathrm{DD}}$ : The driving transistor is in the saturation regime; the load transistor is in the linear regime. The analytical expression for the inverter transfer curve in this region is given by $f_{\mathrm{SL}}\left(V_{\mathrm{in}}\right)(29)$ in the Appendix.

For certain combinations of the transistor parameters, however, $V_{\mathrm{in}, b}<V_{\mathrm{in}, a}$. For example, it can be shown that the depletion-load MOS inverter is operated in this unfavorable regime when $V_{\mathrm{DD}} \leq(\sqrt{n}+1) V_{T}$, assuming identical transistor parameters of the driver and the load $\left(V_{T}=V_{\mathrm{Tl}}=V_{\mathrm{Td}}\right)$. The practical use of this regime is limited, as these conditions will seldom be fulfilled. However, because the focus of this paper is theoretical, and for the sake of completeness, we have taken this operation regime into account.

When $V_{\mathrm{in}, b}<V_{\mathrm{in}, a}$, two more parameters $V_{\mathrm{in}, c}$ and $V_{\mathrm{in}, d}$ need to be defined. $V_{\text {in, }, c}$ represents the input voltage at which the load transistor goes from the saturation into the linear regime, whereas the driving transistor remains in the linear regime. Correspondingly, $V_{\mathrm{in}, d}$ is the input voltage at which the driving transistor goes from the linear into the saturation regime, whereas the load transistor remains in the linear regime. $V_{\mathrm{in}, c}$ and $V_{\mathrm{in}, d}$ are mathematically defined as

$$
\begin{aligned}
V_{\mathrm{out}, c} & =f\left(V_{\mathrm{in}, c}\right) \\
& =f_{\mathrm{LS}}\left(V_{\mathrm{in}, c}\right)=f_{\mathrm{LL}}\left(V_{\mathrm{in}, c}\right) \\
& =V_{\mathrm{Tl}} \\
V_{\mathrm{out}, d} & =f\left(V_{\mathrm{in}, d}\right) \\
& =f_{\mathrm{LL}}\left(V_{\mathrm{in}, d}\right)=f_{\mathrm{SL}}\left(V_{\mathrm{in}, d}\right) \\
& =V_{\mathrm{in}, d}-V_{\mathrm{Td}} .
\end{aligned}
$$

Analytical expressions for $V_{\mathrm{in}, c}$ and $V_{\mathrm{in}, d}$ are found by solving (14)-(19) and are given by (32) and (33) in the Appendix, respectively. It has to be noticed that for a certain set of parameters, either $V_{\mathrm{in}, a}$ and $V_{\mathrm{in}, b}$ or $V_{\mathrm{in}, c}$ and $V_{\mathrm{in}, d}$ provide physically meaningful values. When $V_{\mathrm{in}, a}<V_{\mathrm{in}, b}, V_{\mathrm{in}, c}$ and $V_{\mathrm{in}, d}$ may have a mathematical value, but they have no physical meaning, and, hence, they are not used in our calculations. Conversely, when $V_{\mathrm{in}, b}<V_{\mathrm{in}, a}, V_{\mathrm{in}, a}$ and $V_{\mathrm{in}, b}$ have no meaning, and $V_{\mathrm{in}, c}$ and $V_{\mathrm{in}, d}$ have to be used instead. This is why only $V_{\mathrm{in}, a}$ and $V_{\mathrm{in}, b}$ are shown in Fig. 2.

When $V_{\mathrm{in}, b}<V_{\mathrm{in}, a}$, it can be shown that $V_{\mathrm{in}, c}<V_{\mathrm{in}, d}$, in which case we can distinguish the following operation regimes for the inverter.

1) $0 \leq V_{\mathrm{in}} \leq V_{\mathrm{in}, c}$ : The driving transistor is in the linear regime; the load transistor is in the saturation regime. The analytical expression for the inverter transfer curve in this region is given by $f_{\mathrm{LS}}\left(V_{\mathrm{in}}\right)(26)$ in the Appendix.

2) $V_{\text {in, } c} \leq V_{\text {in }} \leq V_{\text {in, }, d}$ : The driving and the load transistors are both in the linear regime. The analytical expression for the inverter transfer curve in this region is given by $f_{\mathrm{LL}}\left(V_{\mathrm{in}}\right)(28)$ in the Appendix. 


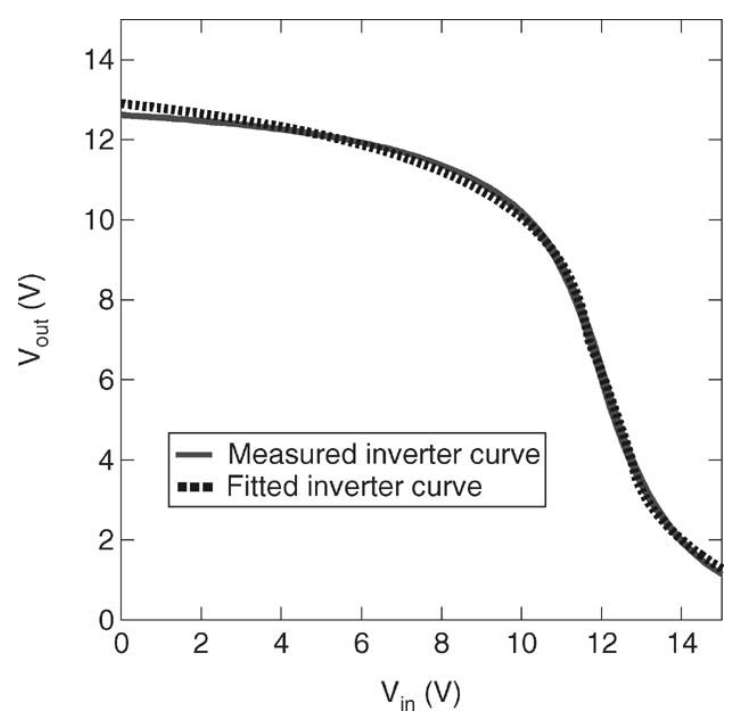

Fig. 4. Calculated and measured inverter transfer curves. Fit parameter values were $V_{\mathrm{Tl}}=4 \mathrm{~V}, V_{\mathrm{Td}}=4.67 \mathrm{~V}, \lambda=0.04 \mathrm{~V}^{-1}$, and $\mu_{l}=0.7 \mu_{d}$.

3) $V_{\mathrm{in}, d} \leq V_{\mathrm{in}} \leq V_{\mathrm{DD}}$ : The driving transistor is in the saturation regime; the load transistor is in the linear regime. The analytical expression for the inverter transfer curve in this region is given by $f_{\mathrm{SL}}\left(V_{\mathrm{in}}\right)(29)$ in the Appendix.

Despite the fact that (6) and (7) completely neglect the subthreshold regime, as well as known OTFT-related issues such as contact effects [17], gate-bias-dependent mobility [18], and bias-stress-dependent threshold voltage [19]-[22], the calculated inverter transfer curves fit very well with the experimentally measured data, as can be verified in Fig. 4. The error made by assuming this very basic transistor model is found to be smaller than $3 \%$.

Experimental data were measured on inverters that were processed on a glass wafer, using $\mathrm{SiO}_{2}$ as the gate dielectric and pentacene as the organic semiconductor. Details about the sample preparation can be found elsewhere [23].

Finally, to calculate the values of the noise margin, we have created an algorithm for maximizing the area of a square that fits in the area between the inverter characteristics $V_{\text {out }}=$ $f\left(V_{\text {in }}\right)$ and $V_{\text {out }}=f^{-1}\left(V_{\text {in }}\right)$.

Using this method, it is possible to simulate the noise margin of an inverter and to investigate the influence of the different parameters on the noise margin. We have simulated how the noise margin is influenced by the field-effect mobility $\mu$, the threshold voltage $V_{T}$, and the output resistance parameter $\lambda$ of the driver and the load OTFTs. Furthermore, we have taken into account statistical variations of the transistor parameters.

\section{RESUlts}

\section{A. Ideal Case}

Fig. 5 shows the calculated noise margin as a function of $V_{T}$, assuming that all transistor parameters of the driver and the load OTFTs are equal. The output resistance parameter $\lambda$ is taken to be zero. These assumptions make this the ideal situation.

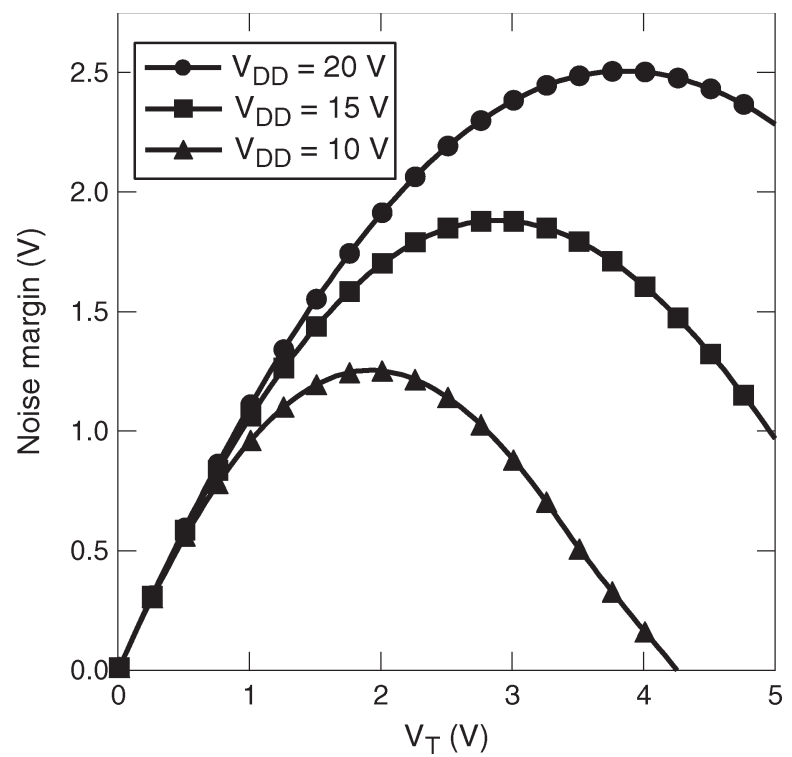

Fig. 5. Influence of the threshold voltage $V_{T}$ on the noise margin for different supply voltages $V_{\mathrm{DD}}$.

As shown in Fig. 5, for any given threshold voltage $V_{T}$, the noise margin can always be increased by increasing the supply voltage. This means that the robustness of the circuit can be improved at the expense of a larger power consumption. Certainly, in practice, this is not the best solution for compensating the effect of unstable transistor parameters. By increasing the supply voltage, effects like degradation and bias stress will occur, and at some point, the device will ultimately break down.

\section{B. Influence of the Output Resistance Parameter $\lambda$ on the Noise Margin}

The first nonideality that was taken into account in our calculations is the output resistance parameter $\lambda$. All parameters $\mu, V_{T}$, and $\lambda$ of the driver and the load OTFTs are assumed to be equal.

Fig. 6 shows the influence of an increase of $\lambda$ on the noise margin. It is clear that even a small but realistic increase of $\lambda$ results in a far worse noise margin. For our own OTFT technology (using pentacene as the organic semiconductor and $\mathrm{SiO}_{2}$ as the gate dielectric), typical extracted values of $\lambda$ are in the range between 0.02 and $0.04 \mathrm{~V}^{-1}$. It can be seen that the noise margin is already decreased by $0.5-1 \mathrm{~V}$, just by accounting realistic values of $\lambda$, as compared to the ideal case in which $\lambda=0$.

This clearly shows that $\lambda$ is at least as important as $\mu$ and $V_{T}$ when it comes to optimizing the stability of OTFTs.

\section{Influence of a Variation of Threshold Voltage $V_{T}$ Between the Driver and the Load OTFT on the Noise Margin}

Despite the fact that a lot of effort has already been spent in improving device uniformity, OTFTs still lack a stable and well-controlled $V_{T}$. Therefore, it is not very realistic to assume 


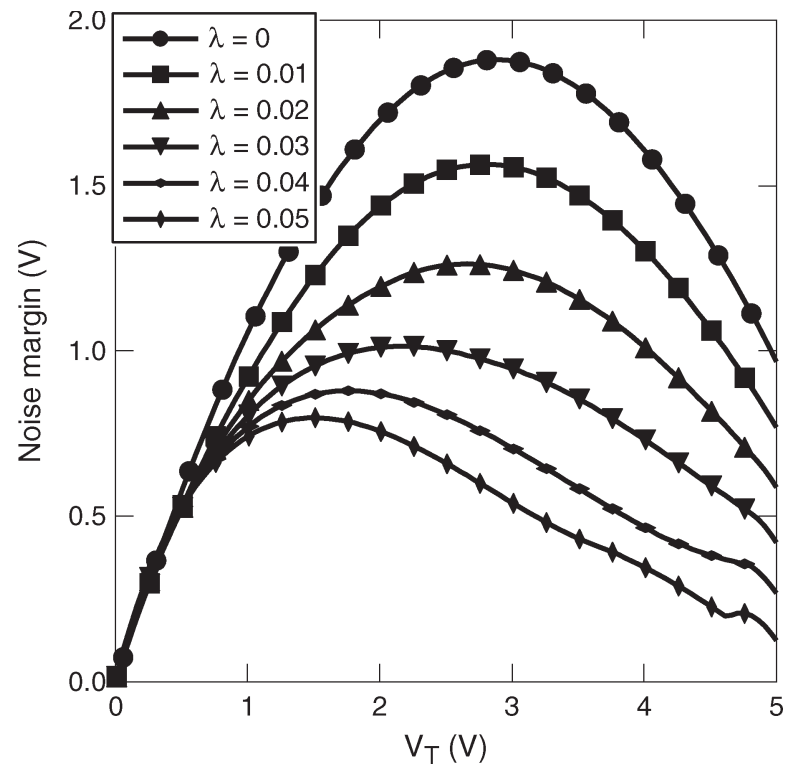

Fig. 6. Influence of the output resistance parameter $\lambda$ on the noise margin for a supply voltage $V_{\mathrm{DD}}$ of $15 \mathrm{~V}$.

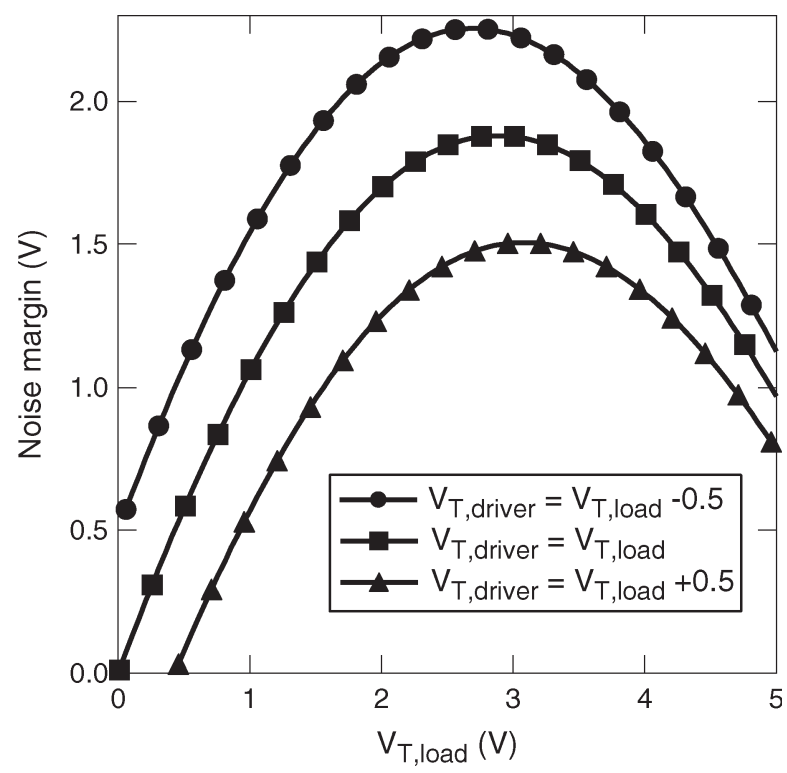

Fig. 7. Influence of a threshold voltage shift $\Delta V_{T}$ on the noise margin for a supply voltage $V_{\mathrm{DD}}$ of $15 \mathrm{~V}(\lambda=0)$.

that the driver and the load OTFTs have identical $V_{T}$. We have simulated the effect of a threshold voltage difference $\Delta V_{T}$ between the driver and the load OTFTs.

Fig. 7 shows the influence of a $\Delta V_{T}$ of $\pm 0.5 \mathrm{~V}$. It is easily seen that a shift in $V_{T}$ may turn out to be either advantageous or disadvantageous. The noise margin increases when the driver OTFT has a lower $V_{T}$ than that of the load OTFT; on the other hand, when $V_{\mathrm{Td}}$ is larger than $V_{\mathrm{Tl}}$, the noise margin decreases. Controlling the threshold voltage of the driver and the load OTFTs, thereby influencing the noise margin, may be technologically realized by a patterned growth of the organic semiconductor [24].

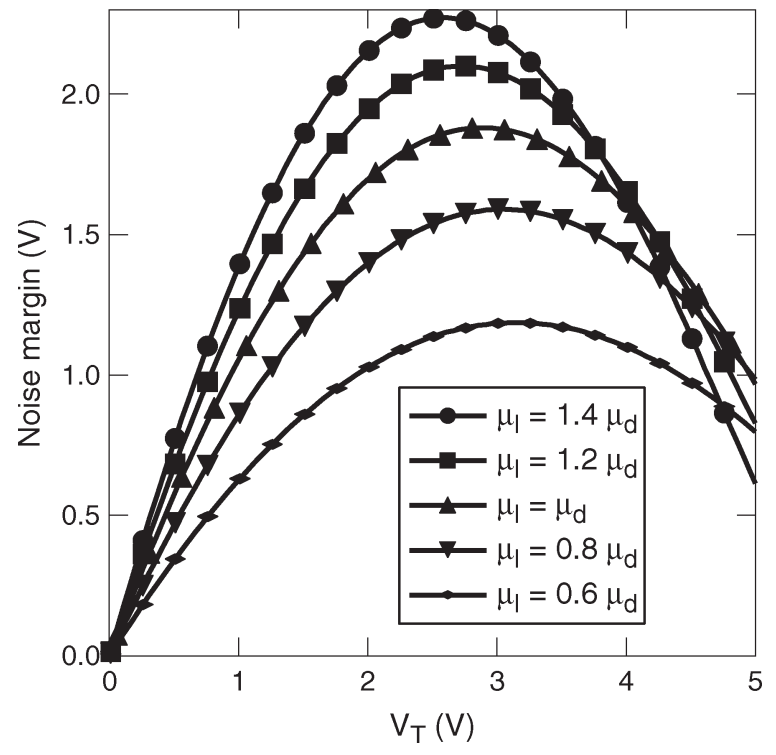

Fig. 8. Influence of mobility variations on the noise margin of an inverter. The curves are given for a relative mobility variation of $\pm 20 \%$ and $\pm 40 \%$ between the driver and the load OTFT. $V_{\mathrm{DD}}=15 \mathrm{~V}$ and $\lambda=0$.

\section{Influence of a Variation of the Field-Effect Mobility $\mu$ Between the Driver and the Load OTFTs on the Noise Margin}

The field-effect mobility $\mu$ is another transistor parameter that is known to be distributed over the wafer area. Very much like the case of variations in $V_{T}$, variations of the mobility of the driver and the load OTFTs will have an influence on the noise margin of the inverter.

Fig. 8 shows how the noise margin is affected by relative mobility changes of $\pm 20 \%$ and $\pm 40 \%$ between the driver and the load OTFTs. Like in the case for a $\Delta V_{T}$ between the driver and the load transistors, different mobilities can have either a beneficial or a detrimental influence on the noise margin. The noise margin increases when the mobility of the load OTFT is larger than the mobility of the driver OTFT. As for the case of $\Delta V_{T}$, a patterned growth of the organic semiconductor may lead to a controlled variation in the field-effect mobility $\mu$ between the driver and the load OTFTs [24], resulting in an improved noise margin.

\section{E. Influence of a Variation of the Threshold Voltage $V_{T}$ Between Two Successive Inverter Stages on the Noise Margin}

When considering a chain of inverters (for example, in a ring oscillator), it is important that the logic levels of two successive inverter stages are matched. The transfer curve of an inverter can shift because of, for example, temperature or process variations. In a conventional Si CMOS technology, transistor parameters are stable and well defined, thus, the contribution of process variations can be neglected. Therefore, in Si CMOS technology, the noise margin, as defined earlier, is truly a margin that only has to account for noise sources: as long as the noise voltage at the input of a logic gate is smaller than the noise margin of the circuit, the circuit will function properly. 


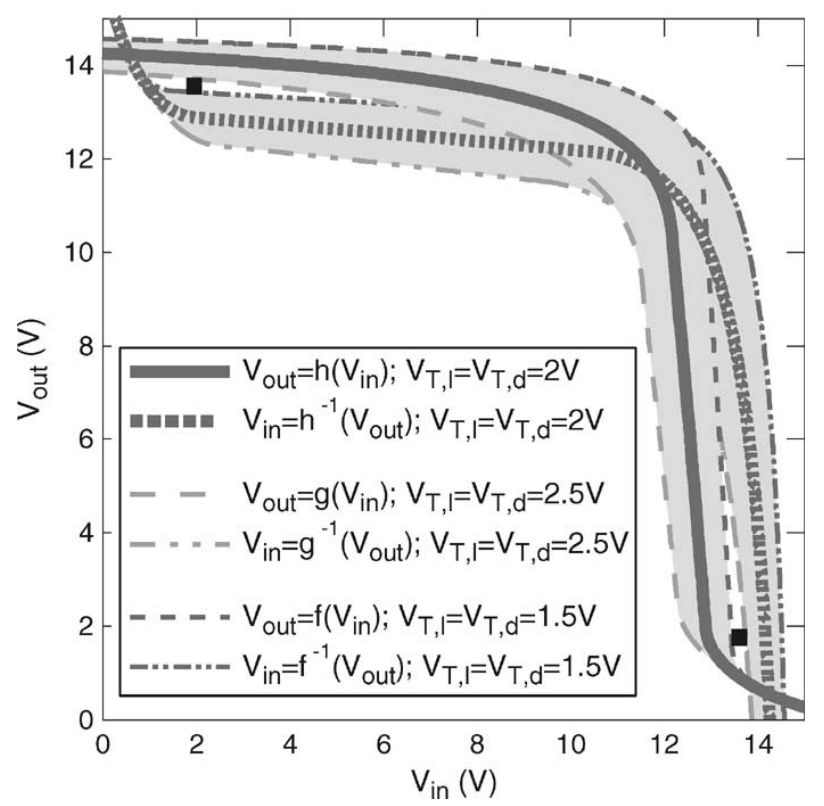

Fig. 9. Family of transfer curves for $V_{\mathrm{DD}}=15 \mathrm{~V}$ and $\lambda=0.02 \mathrm{~V}^{-1}$. Three transfer curves are shown: $V_{T}=1.5,2$, and $2.5 \mathrm{~V}$. The black square represents the worst case noise margin resulting from this set of curves.

In OTFT technology, however, the transistor parameters are typically distributed over a relatively broad range [25]. For our processing technology, as described in [23], we obtain a standard deviation $\sigma_{V_{T}}=0.6 \mathrm{~V}$ for the distribution of $V_{T}$ on one substrate. As a comparison, in a typical $0.13-\mu$ CMOS technology, the threshold voltage is allowed to vary less than $\pm 100 \mathrm{mV}$. This shows that the standard deviation of $V_{T}$ in an organic technology is significantly larger than that of a recent Si CMOS technology.

Inverters based on OTFTs with variable transistor parameters lead to a family of transfer curves. Fig. 9 shows a set of inverter transfer curves for an average $V_{T}$ of $2 \mathrm{~V}$ and two curves for a $V_{T}$ of 1.5 and $2.5 \mathrm{~V}$, respectively. The shaded area then represents all possible transfer curves with threshold voltages in the range of $2 \pm 0.5 \mathrm{~V}$, assuming there is no variation in the other transistor parameters.

From the graphical representation in Fig. 9, it can be easily understood that the noise margin will be heavily influenced by the distribution of $V_{T}$.

As a worst case, we have calculated the noise margin of a circuit consisting of an inverter $M$ with a transfer curve $f\left(V_{\text {in }}\right)$, where $V_{T, M}=V_{T, \text { avg }}-\Delta V_{T}$, followed by an inverter $M+1$ with a transfer curve $g\left(V_{\text {in }}\right)$, where $V_{T, M+1}=V_{T \text {,avg }}+\Delta V_{T}$. Within the individual inverters $M$ and $M+1$, the driver and the load transistors have the same transistor parameters $\mu, V_{T}$, and $\lambda$.

Instead of calculating the noise margin by maximizing the area of a square between $f\left(V_{\text {in }}\right)$ and $f^{-1}\left(V_{\text {in }}\right)$, we now use the area between $f\left(V_{\text {in }}\right)$ and $g^{-1}\left(V_{\text {in }}\right)$. Two different squares of maximum area can be found between $f\left(V_{\text {in }}\right)$ and $g^{-1}\left(V_{\text {in }}\right)$, corresponding to $N M_{H}$ and $N M_{L}$ of the system of the two successive inverters. When $\Delta V_{T}=0, N M_{H}=N M_{L}$; however, when $\Delta V_{T} \neq 0, N M_{H} \neq N M_{L}$. The noise margin of the system of the two successive inverters $M$ and $M+1$ is defined as the worst case noise margin, i.e., $N M=\min \left(N M_{H}, N M_{L}\right)$.

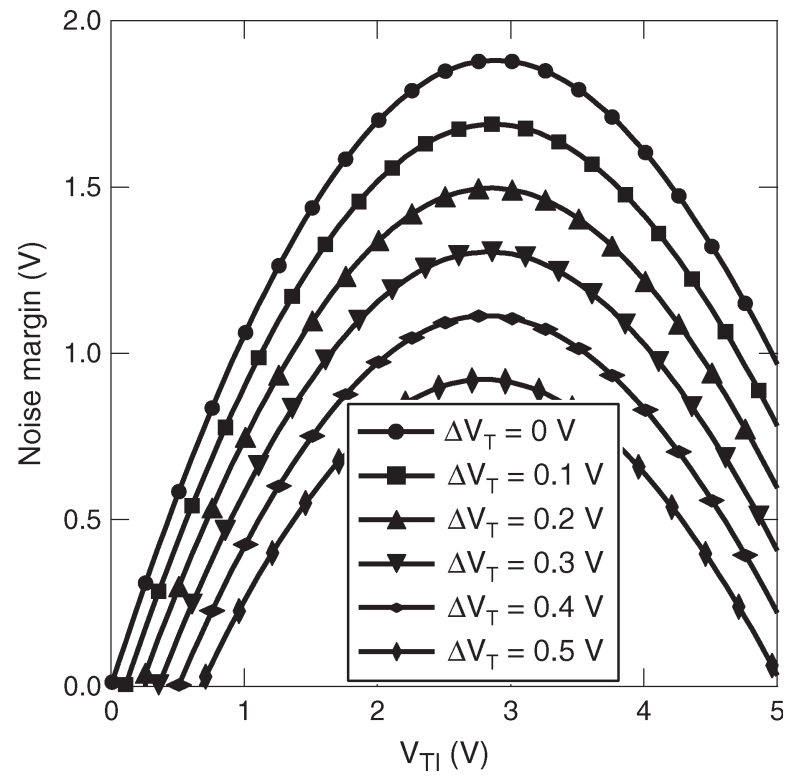

Fig. 10. Worst case calculation of the noise margin, taking into account a difference in $V_{T}$ between two successive inverter stages $\left(V_{T, M+1}-V_{T, M}=\right.$ $\left.2 \Delta V_{T}\right) . V_{\mathrm{DD}}=15 \mathrm{~V}$ and $\lambda=0$. We assumed that the $V_{T}$ of the driver and the load OTFT are equal in each individual inverter.

Fig. 10 shows this worst case noise margin as a function of the average $V_{T}$, using the parameter $\Delta V_{T}$, where $\Delta V_{T}$ is defined as

$$
\Delta V_{T}=\frac{1}{2}\left(V_{T, M+1}-V_{T, M}\right) .
$$

An increase in $\Delta V_{T}$ clearly results in a worse noise margin. Although inverters with no statistical variation show a noise margin of $1.8 \mathrm{~V}$ with the parameters used, this worst case calculation for a $\Delta V_{T}=0.5 \mathrm{~V}$ results in a noise margin of about $1 \mathrm{~V}$ less.

These results clearly demonstrate that the uniformity of $V_{T}$ over the wafer is crucial in order to produce circuits using a substantial number of OTFTs. This statement will be quantified Section V.

\section{ANALYSis}

In this section, we will analyze the influence of the distribution of $V_{T}$ on the worst case yield, as discussed earlier in case $E$, i.e., for a statistical distribution of the threshold voltage $V_{T}$ between two successive inverter stages. We assume that the driver and the load transistors of each individual inverter have identical $\mu, V_{T}$, and $\lambda$. Further, this yield analysis is made for a fairly realistic parameter set: $V_{\mathrm{DD}}=15 \mathrm{~V}$; average $V_{T}=\mu_{V_{T}}=3 \mathrm{~V}$; and $\lambda=0.02 \mathrm{~V}^{-1}$.

With the average threshold voltage $\mu_{V_{T}}$ kept constant, the worst case noise margin of a system of two successive inverters varies linearly with $\Delta V_{T}$, as can be confirmed in Fig. 10. It was found that for the parameter set used in the analysis, this dependence is given by

$$
\begin{aligned}
N M & =\min \left(N M_{H}, N M_{L}\right) \\
& =1.06-2.26 \cdot\left|\Delta V_{T}\right| .
\end{aligned}
$$


Equation (22) shows that the maximum noise margin is $1.06 \mathrm{~V}$, which is reached when $\Delta V_{T}=0$. For $\Delta V_{T} \neq 0$, either $N M_{H}$ or $N M_{L}$ is larger than this maximum noise margin. At the same time, however, the corresponding $N M_{L}$ or $N M_{H}$ decreases. This lower noise margin obviously determines the worst case noise margin. This reasoning justifies the use of $\left|\Delta V_{T}\right|$ rather than simply $\Delta V_{T}$ in (22).

Inasmuch as the dependence of $N M$ on $\Delta V_{T}$ is essentially linear, a Gaussian distribution of $V_{T}$ will lead to a Gaussian distribution of the noise margin [26]. The mean of this noise margin distribution is located at $\mu_{\mathrm{NM}}=1.06 \mathrm{~V}$. As the noise margin is calculated as the difference of two normally distributed variables $\left[f\left(V_{\text {in }}\right)\right.$ and $\left.g^{-1}\left(V_{\text {in }}\right)\right]$ with the same standard deviation, the standard deviation of the noise margin distribution is given by $\sigma_{\mathrm{NM}}=\sqrt{2} \cdot 2.26 \cdot \sigma_{V_{T}}$, where $\sigma_{V_{T}}$ is the standard deviation of the distribution of $V_{T}$.

However, the presence of $\left|\Delta V_{T}\right|$ in (22) implies that the noise margin can never be higher than $\mu_{\mathrm{NM}}$. Therefore, the right half of the Gaussian probability density function $\left(N M>\mu_{\mathrm{NM}}\right)$ is mirrored on the left half. Due to the symmetry of the normal density function, this operation doubles the probability density function for $N M<\mu_{\mathrm{NM}}$ [27]. This is graphically illustrated in Fig. 11.

In our analysis, we have defined a defective pair of inverters $M$ and $M+1$ as one that results in $N M<0$. Hence, the defect probability $P_{D}$ of an inverter pair is easily found by calculating the normal cumulative distribution function $D(x)$ of the noise margin for $N M<0$ as

$$
\begin{aligned}
P_{D} & =2 \cdot D(0) \\
& =2 \cdot \int_{-\infty}^{0} \frac{1}{\sqrt{2 \pi} \sigma_{\mathrm{NM}}} \exp \left(-\frac{\left(x-\mu_{\mathrm{NM}}\right)^{2}}{2 \sigma_{\mathrm{NM}}^{2}}\right) d x
\end{aligned}
$$

as shown in Fig. 11. As indicated before, the prefactor 2 in (23) and (24) needs to be included because of the doubled Gaussian probability density function for $N M<\mu_{\mathrm{NM}}$.

The defect probability $P_{D}$ is commonly called $\lambda$ in statistics; however, to avoid any confusion with the output resistance parameter $\lambda$ used in this manuscript, we have chosen to represent the defect probability by $P_{D}$. For small values of $P_{D}$, the yield can be approximated by the Poisson distribution [28] defined by

$$
Y=e^{-N \cdot P_{D}}
$$

in which $N$ is the number of inverters used in the circuit. The result is shown in Fig. 12. Much more than the simple noise margin analysis, this plot clearly shows the tremendous influence of the distribution of $V_{T}$ on the circuit yield.

\section{CONCLUSION}

We have calculated the analytical expressions for the transfer curve of an inverter based on OTFTs. The calculated results fit well with the experimentally measured inverter curves. Using

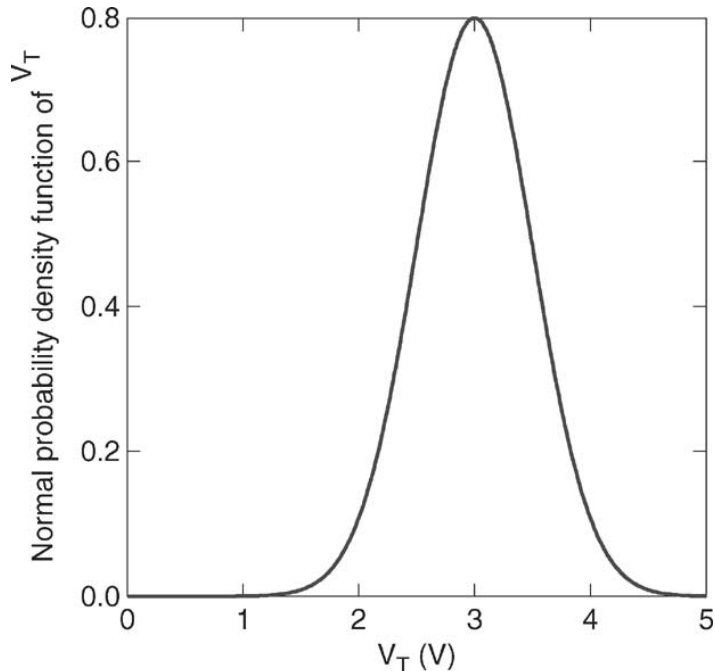

(a)

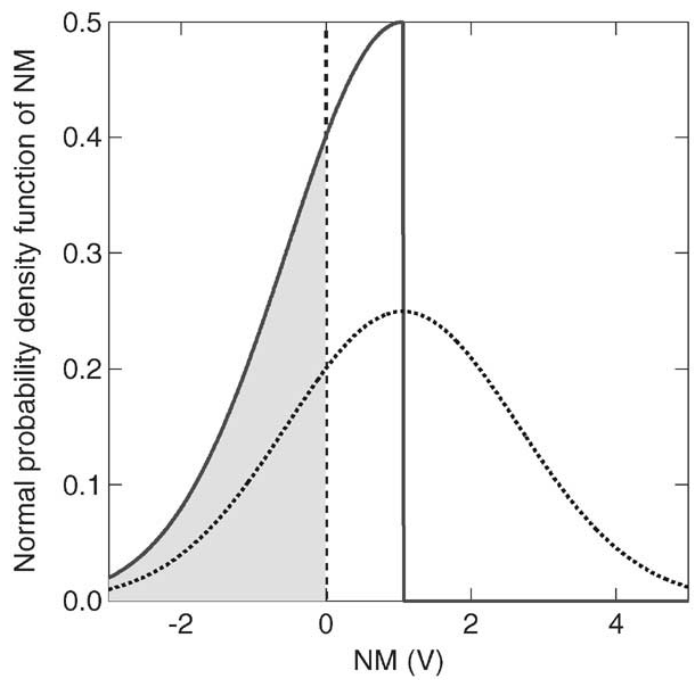

(b)

Fig. 11. (a) Gaussian probability density function of $V_{T}$ with mean $\mu_{V_{T}}=$ $3 \mathrm{~V}$ and $\sigma_{V_{T}}=0.5 \mathrm{~V}$. (b) Dotted line represents the probability density function of $N M$ when (22) would simply read $N M=1.06-2.26 \cdot \Delta V_{T}$ (Gaussian with $\mu_{\mathrm{NM}}=1.06 \mathrm{~V}$ and $\sigma_{\mathrm{NM}}=\sqrt{2} \cdot 2.26 \cdot \sigma_{V_{T}}$ ). The solid line is the actual Gaussian probability density function of $N M$, using $\left|\Delta V_{T}\right|$ rather than $\Delta V_{T}$ in (22). The gray area illustrates the defect probability $(N M<0)$, as calculated in (24).

these analytical expressions, we have investigated the influence of the transistor parameters $V_{T}, \mu$, and $\lambda$ of the driver and the load OTFTs on the noise margin of inverters, which is a wellknown figure for the robustness of digital circuits. The noise margin was calculated using the MEC.

The initial conclusion is that, given any set of transistor parameters, the noise margin can always be increased by increasing the supply voltage $V_{\mathrm{DD}}$. However, increasing $V_{\mathrm{DD}}$ should not be used for compensating an intrinsic lack of robustness.

Higher values of the output resistance parameter $\lambda$ proved to be detrimental for the noise margin. This shows that $\lambda$ is an important parameter for realizing stable circuits.

Variations of $V_{T}$ and $\mu$ between the driver and the load OTFTs of the same inverter can result in either an improved or a reduced noise margin. 


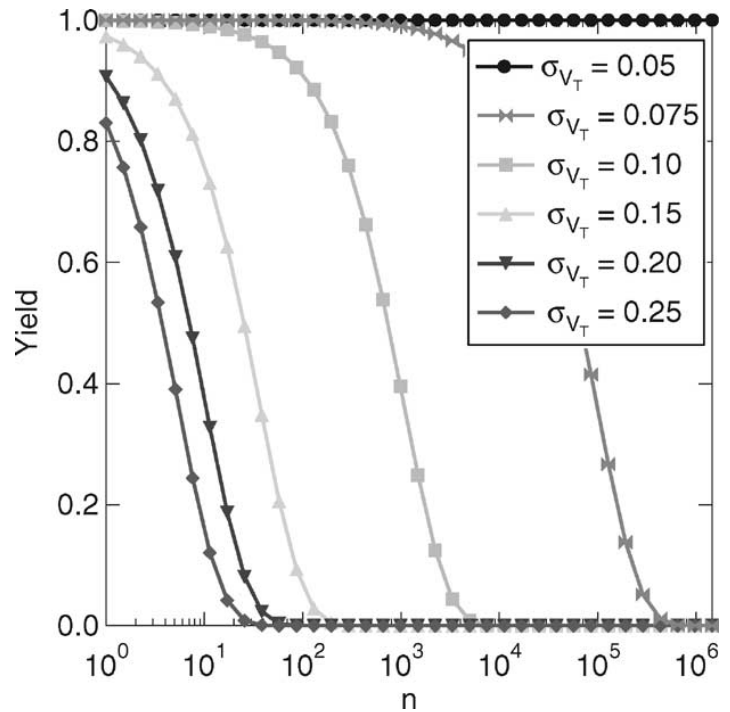

Fig. 12. Worst case calculation of the circuit yield as a function of the number of inverters, calculated for $V_{\mathrm{DD}}=15 \mathrm{~V}, V_{T}=3 \mathrm{~V}$, and $\lambda=0.02 \mathrm{~V}^{-1}$.

Taking into account the fact that $V_{T}$ is statistically distributed over the wafer, the worst case noise margin is clearly reduced with higher standard deviations of the parameter distribution. This statement was quantified by calculating the expected worst case circuit yield as a function of the circuit complexity, for different values of the standard deviation of $V_{T}$.

The earlier analysis is simplified in the sense that it assumes that each inverter consists of a driver and a load transistor that have identical transistor parameters. A more refined model could, for example, consider a local threshold voltage variation within an individual inverter, in addition to the global threshold voltage spread in the circuit. However, such analysis goes beyond the scope of this paper.

\section{APPENDIX \\ ANALYTICAL EXPRESSIONS FOR THE TRANSITION POINTS AND THE INVERTER TRANSFER CURVE}

The output voltage $V_{\text {out }}$ is obtained as an analytical expression of the input voltage $V_{\text {in }}$ using the following conditions:

1) When the driver is in the linear regime, and the load is in the saturation regime (i.e., $0 \leq V_{\text {in }} \leq V_{\text {in }, a}$ or $0 \leq V_{\text {in }} \leq$ $\left.V_{\mathrm{in}, c}\right), V_{\text {out }}$ is defined by (26), shown at the bottom of the page.

2) When the driver and the load are both in the saturation regime (i.e., $V_{\mathrm{in}, a} \leq V_{\mathrm{in}} \leq V_{\mathrm{in}, b}$ ), $V_{\text {out }}$ is defined as (27), shown at the bottom of the page.

3) When the driver and the load are both in the linear regime (i.e., $V_{\mathrm{in}, c} \leq V_{\mathrm{in}} \leq V_{\mathrm{in}, d}$ ), $V_{\text {out }}$ is defined by (28), shown at the bottom of the page.

4) When the driver is in the saturation regime and the load is in the linear regime (i.e., $V_{\mathrm{in}, b} \leq V_{\mathrm{in}} \leq V_{\mathrm{DD}}$ or $V_{\mathrm{in}, d} \leq$ $\left.V_{\text {in }} \leq V_{\mathrm{DD}}\right), V_{\text {out }}$ is defined by (29), shown at the bottom of the page.

The transition points $V_{\mathrm{in}, a}, V_{\mathrm{in}, b}, V_{\mathrm{in}, c}$, and $V_{\mathrm{in}, d}$ are given by (30)-(33), shown at the top of the next page.

In these equations, $V_{\mathrm{DD}}$ is the supply voltage, $V_{\mathrm{Td}}$ is the threshold voltage of the driver, $V_{\mathrm{Tl}}$ is the threshold voltage of the load, $\lambda$ is the output resistance parameter, and $n$ is the ratio of the $W / L$ of the load and the $W / L$ of the driver.

$$
\begin{aligned}
V_{\text {out }} & =f_{\mathrm{LS}}\left(V_{\mathrm{in}}\right) \\
& =V_{\mathrm{in}}-V_{\mathrm{Td}}-\frac{n \lambda V_{\mathrm{Tl}}^{2}}{2}+\frac{1}{2} \sqrt{\left(2 V_{\mathrm{Td}}+n \lambda V_{\mathrm{Tl}}^{2}-2 V_{\mathrm{in}}\right)^{2}+4\left(V_{\mathrm{DD}}\left(V_{\mathrm{DD}}+2 V_{\mathrm{Td}}-2 V_{\mathrm{in}}\right)+n V_{\mathrm{Tl}}^{2}\left(\lambda V_{\mathrm{Tl}}-1\right)\right)}
\end{aligned}
$$

$$
V_{\text {out }}=f_{\mathrm{SS}}\left(V_{\mathrm{in}}\right)=\frac{\left(V_{\mathrm{DD}}+V_{\mathrm{Td}}-V_{\mathrm{in}}\right)^{2}\left(1+\lambda V_{\mathrm{in}}-\lambda V_{\mathrm{Td}}\right)-n V_{\mathrm{Tl}}^{2}+n \lambda V_{\mathrm{Tl}}^{3}}{\lambda\left(\left(V_{\mathrm{DD}}+V_{\mathrm{Td}}-V_{\mathrm{in}}\right)^{2}+n V_{\mathrm{Tl}}^{2}\right)}
$$

$$
\begin{aligned}
V_{\mathrm{out}} & =f_{\mathrm{LL}}\left(V_{\mathrm{in}}\right) \\
& =\frac{V_{\mathrm{Td}}+n V_{\mathrm{Tl}}-V_{\mathrm{in}}-\sqrt{\left(V_{\mathrm{Td}}+n V_{\mathrm{Tl}}-V_{\mathrm{in}}\right)^{2}-(n-1) V_{\mathrm{DD}}\left(V_{\mathrm{DD}}+2 V_{\mathrm{Td}}-2 V_{\mathrm{in}}\right)}}{n-1}
\end{aligned}
$$

$$
\begin{aligned}
V_{\mathrm{out}}= & f_{\mathrm{SL}}\left(V_{\mathrm{in}}\right) \\
= & \frac{\lambda\left(V_{\mathrm{DD}}+V_{\mathrm{Td}}-V_{\mathrm{in}}\right)^{2}+2 n V_{\mathrm{Tl}}}{2 n} \\
& -\frac{1}{2 n} \sqrt{\lambda^{2}\left(V_{\mathrm{DD}}+V_{\mathrm{Td}}-V_{\mathrm{in}}\right)^{4}-4 n\left(V_{\mathrm{DD}}+V_{\mathrm{Td}}-V_{\mathrm{in}}\right)^{2}\left(1+\lambda\left(V_{\mathrm{in}}-V_{\mathrm{Td}}-V_{\mathrm{Tl}}\right)\right)+4 n^{2} V_{\mathrm{Tl}}^{2}}
\end{aligned}
$$




$$
\begin{aligned}
& V_{\mathrm{in}, a}=V_{\mathrm{DD}}+V_{\mathrm{Td}}+\frac{n \lambda V_{\mathrm{Tl}}^{2}}{2}-\sqrt{n} V_{\mathrm{Tl}} \sqrt{1+\lambda V_{\mathrm{DD}}-\lambda V_{\mathrm{Tl}}+\frac{1}{4} n \lambda^{2} V_{\mathrm{Tl}}^{2}} \\
& V_{\mathrm{in}, b}=\frac{2}{3} V_{\mathrm{DD}}+V_{\mathrm{Td}}+\frac{V_{\mathrm{Tl}}}{3}-\frac{1}{3 \lambda}+\frac{2}{3 \lambda} \cos \left(\frac{\pi}{3}+\frac{1}{3} \arccos \left(1-\frac{27 n \lambda^{2} V_{\mathrm{Tl}}^{2}}{2\left(1+\lambda V_{\mathrm{DD}}-\lambda V_{\mathrm{Tl}}\right)^{3}}\right)\right)\left(1+\lambda V_{\mathrm{DD}}-\lambda V_{\mathrm{Tl}}\right) \\
& V_{\mathrm{in}, c}=\frac{V_{\mathrm{DD}}^{2}+2 V_{\mathrm{DD}} V_{\mathrm{Td}}-V_{\mathrm{Tl}}\left(2 V_{\mathrm{Td}}+(n+1) V_{\mathrm{Tl}}\right)}{2\left(V_{\mathrm{DD}}-V_{\mathrm{Tl}}\right)} \\
& V_{\mathrm{in}, d}=\frac{V_{\mathrm{DD}}+V_{\mathrm{Td}}+n V_{\mathrm{Td}}+n V_{\mathrm{Tl}}-\sqrt{n^{2} V_{\mathrm{Tl}}^{2}+2 n V_{\mathrm{DD}} V_{\mathrm{Tl}}-n V_{\mathrm{DD}}^{2}}}{n+1}
\end{aligned}
$$

\section{ACKNOWLEDGMENT}

The authors thank P. Roussel for his collaboration with the yield analysis.

\section{REFERENCES}

[1] C. F. Hill, "Definitions of noise margin in logic systems," Mullard Tech. Commun., no. 89, pp. 239-245, Sept. 1967.

[2] — "Noise margin and noise immunity in logic circuits," Microelectronics, vol. 1, no. 4, pp. 16-21, Sep. 1968.

[3] E. Cantatore, C. M. Hart, M. Digioia, G. H. Gelinck, T. C. T. Geuns, H. E. A. Huitema, L. R. R. Schrijnemakers, E. van Veenendaal, and D. M. de Leeuw, "Circuit yield of organic integrated electronics," in Proc. ISSCC, San Francisco, CA, 2003, pp. 382-383.

[4] E. Cantatore and E. J. Meijer, "Transistor operation and circuit performance in organic electronics," in Proc. ESSCIRC, Estoril, Portugal, 2003, pp. 29-36.

[5] C. A. Lee, S. H. Jin, K. D. Jung, J. D. Lee, and B.-G. Park, "Full-swing pentacene organic thin-film transistor inverter with enhancement-mode driver and depletion-mode load," in Proc. DRC, Notre Dame, IN, 2004, pp. 181-182.

[6] J. R. Hauser, "Noise margin criteria for digital logic circuits," IEEE Trans. Educ., vol. 36, no. 4, pp. 363-368, Nov. 1993.

[7] J. S. Yuan and L. Yang, "Teaching digital noise and noise margin issues in engineering education," IEEE Trans. Educ., vol. 48, no. 1, pp. 162-168, Feb. 2005.

[8] S. De Vusser, S. Steudel, K. Myny, J. Genoe, and P. Heremans, "A 2 $\mathrm{V}$ organic complementary inverter," in Proc. ISSCC, San Francisco, CA, 2006, pp. 282-283.

[9] B. Crone, A. Dodabalapur, Y.-Y. Lin, R. W. Filas, Z. Bao, A. LaDuca, R. Sarpeshkar, H. E. Katz, and W. Li, "Large-scale complementary integrated circuits based on organic transistors," Nature, vol. 403, no. 6769, pp. 521-523, Feb. 2000

[10] H. Klauk, M. Halik, U. Zschieschang, F. Eder, D. Rohde, G. Schmid, and C. Dehm, "Flexible organic complementary circuits," IEEE Trans. Electron Devices, vol. 52, no. 4, pp. 618-622, Apr. 2005.

[11] D. J. Gundlach, K. P. Pernstich, G. Wilckens, M. Grüter, S. Haas, and B. Batlogg, "High mobility n-channel organic thin-film transistors and complementary inverters," J. Appl. Phys., vol. 98, no. 6, p. 064502, Sep. 2005.

[12] H. Klauk, D. J. Gundlach, and T. N. Jackson, "Fast organic thin-film transistor circuits," IEEE Electron Device Lett., vol. 20, no. 6, pp. 289291, Jun. 1999

[13] G. H. Gelinck, T. C. T. Geuns, and D. M. de Leeuw, "High-performance all-polymer integrated circuits," Appl. Phys. Lett., vol. 77, no. 10, pp. 1487-1489, Sep. 2000.

[14] H. Klauk, M. Halik, U. Zschieschang, F. Eder, G. Schmid, and C. Dehm, "Pentacene organic transistors and ring oscillators on glass and on flexible polymeric substrates," Appl. Phys. Lett., vol. 82, no. 23, pp. 4175-4177, Jun. 2003.

[15] O. J. McCarthy, MOS Device and Circuit Design. New York: Wiley, 1982.

[16] S. M. Sze, Physics of Semiconductor Devices. New York: Wiley, 1981.
[17] R. A. Street and A. Salleo, "Contact effects in polymer transistors," Appl. Phys. Lett., vol. 81, no. 15, pp. 2887-2889, Oct. 2002.

[18] G. Horowitz, P. Lang, M. Mottaghi, and H. Aubin, "Extracting parameters from the current-voltage characteristics of organic field-effect transistors," Adv. Funct. Mater., vol. 14, no. 11, pp. 1069-1074, 2004.

[19] D. Knipp, R. A. Street, A. Volkel, and J. Ho, "Pentacene thin-film transistors on inorganic dielectrics: Morphology, structural properties, and electronic transport," J. Appl. Phys., vol. 93, no. 1, pp. 347-355, Jan. 2003.

[20] J. Genoe, S. Steudel, S. De Vusser, S. Verlaak, D. Janssen, and P. Heremans, "Bias stress in pentacene transistors measured by four probe transistor structures," in Proc. ESSDERC, Leuven, Belgium, 2004 pp. 413-416.

[21] H. L. Gomes, P. Stallinga, F. Dinelli, M. Murgia, F. Biscarini, D. M de Leeuw, T. Muck, J. Geurts, L. W. Molenkamp, and V. Wagner, "Bias-induced threshold voltages shifts in thin-film organic transistors," Appl. Phys. Lett., vol. 84, no. 16, pp. 3184-3186, Apr. 2004.

[22] R. A. Street, A. Salleo, and M. L. Chabinyc, "Bipolaron mechanism for bias-stress effects in polymer transistors," Phys. Rev. B, Condens. Matter, vol. 68 , no. 8, p. 085316 , Aug. 2003.

[23] S. De Vusser, S. Steudel, K. Myny, J. Genoe, and P. Heremans, "High-performance low voltage organic thin-film transistors," in Proc. Mater. Res. Society Symp. 870E-Giant-Area Electron. on Nonconventional Substrates, M.S. Shur, P. Wilson, and M. Stutzmann, Eds. PA: Warrendale, 2005, pp. H1.4.1-H1.4.5.

[24] S. Steudel, D. Janssen, S. Verlaak, J. Genoe, and P. Heremans, "Patterned growth of pentacene," Appl. Phys. Lett., vol. 85, no. 23, pp. 5550-5552, Dec. 2004.

[25] D. M. de Leeuw, G. H. Gelinck, T. C. T. Geuns, E. van Veenendaal, E. Cantatore, and B. H. Huisman, "Polymeric integrated circuits: Fabrication and first characterization," in IEDM Tech. Dig., San Francisco, CA, 2002, pp. 293-296.

[26] J. K. Patel and C. B. Read, Handbook of the Normal Distribution. New York: Marcel Dekker, 1982.

[27] P. J. Roussel, R. Degraeve, G. V. Van den Bosch, B. Kaczer, an G. Groeseneken, "Accurate and robust noise-based trigger algorithm for soft breakdown detection in ultrathin gate dielectrics," IEEE Trans. Device Mater. Rel., vol. 1, no. 2, pp. 120-127, Jun. 2001.

[28] A. Papoulis, Probability, Random Variables, and Stochastic Processes. New York: McGraw-Hill, 1984.

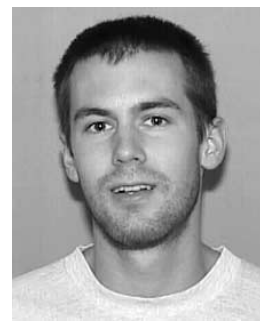

Stijn De Vusser (S'05) received the M.S. degree in electrical engineering from Katholieke Universiteit Leuven, Leuven, Belgium, in 2001. He is currently working toward the Ph.D. degree in electrical engineering at the Interuniversity Microelectronics Center (IMEC), Leuven, Belgium, working in the field of OTFT technology.

$\mathrm{He}$ is also with the Electrical Engineering Department, Katholieke Universiteit Leuven. 


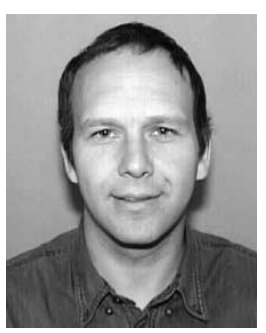

Jan Genoe (S'87-M'02) received the M.S. degree in electrical engineering and the Ph.D. degree in applied sciences from Katholieke Universiteit Leuven, Leuven, Belgium, in 1988 and 1994, respectively.

$\mathrm{He}$ is a Lecturer at Katholieke Hogeschool Limburg, Diepenbeek, Belgium, and a Senior Researcher at the Polymer and Molecular Electronics (PME) Group, Interuniversity Microelectronics Center (IMEC), Leuven, Belgium.

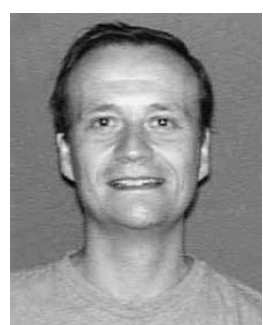

Paul Heremans received the Ph.D. degree in applied sciences from Katholieke Universiteit Leuven, Leuven, Belgium, in 1990.

From 1984 to 1990, he was a Research Assistant and a Senior Research Assistant at the Belgian Fund for Scientific Research. He is presently the Group Leader of the Organic Electronics Group, Interuniversity Microelectronics Center (IMEC), Leuven, Belgium. He is also a Professor at the Electrical Engineering Department, Katholieke Universiteit Leuven. 